\title{
Kultur und Wissen digital vermitteln - Stand und Perspektiven der Deutschen Digitalen Bibliothek - ein Überblick
}

DOI 10.1515/bfp-2017-0008

Zusammenfassung: Die Deutsche Digitale Bibliothek (DDB) schafft über das Internet freien Zugang zum kulturellen und wissenschaftlichen Erbe Deutschlands. Sie vernetzt die digitalen Angebote deutscher Museen, Bibliotheken, Archive, Denkmalpflegeeinrichtungen, Mediatheken und Forschungsinstitutionen und macht sie kostenfrei online zugänglich. Ihre Sammlungen sind so über ein zentrales nationales Portal auffindbar. Gestartet als ein gemeinsam von Bund und Ländern gefördertes Projekt, hat die DDB ihre Aufbauphase inzwischen erfolgreich durchlaufen. Mit mehr als 20 Mio. nachgewiesenen Objekten von über 280 Partnern, einer modernen und attraktiven Benutzeroberfläche, einer aktiven Lieferbeziehung zu Europeana und einer zunehmenden Nutzung und Wahrnehmung in der deutschen und europäischen Öffentlichkeit wurden wesentliche Ziele der Aufbauphase erreicht. Der Artikel berichtet über die Idee, die Umsetzung sowie den bisher mit der DDB erreichten Stand. Des Weiteren informiert der Beitrag über die geplanten Entwicklungen in den nächsten Jahren und die damit einhergehenden Herausforderungen.

Schlüsselwörter: Spartenübergreifendes, interdisziplinäres Zugangsportal zum kulturellen Erbe Deutschlands; Strategie der DBB 2015-2020; klare Perspektive mit langfristiger Finanzierung als dauerhafte staatliche Aufgabe

\section{Current Status and Perspectives of Deutsche Digitale Bibliothek - an Overview}

\begin{abstract}
The Deutsche Digitale Bibliothek (DDB) networks the digitized collections of German museums, archives, research institutes, print, and multimedia libraries and monument preservation organizations, thereby providing unrestricted online access to German cultural and scientific heritage at no charge to the users. Started as a jointly funded federal and state project, the DDB has completed its developing stage successfully now. With more than 20 million objects provided by more than 280 part-
\end{abstract}

*Kontaktperson: Frank Frischmuth, f.frischmuth@hv.spk-berlin.de ners, a modern user interface, an active supplier relationship to Europeana and an increasing use and recognition in the German and European public, key objectives have been achieved. The article informs about the ideas behind the project as well as the achievements and results reached so far. Furthermore, the contribution is an outlook on future challenges and coming up trends beyond 2015.

Keywords: Inter-divisional, interdisciplinary access to Germany's cultural heritage; strategy of DBB 2015-2020; clear perspective and long-term funding with federal and state engagement

\section{Einleitung}

Idee und Anstoß für eine Deutsche Digitale Bibliothek (DDB) gehen auf einen gemeinsamen Brief von sechs europäischen Staats- und Regierungschefs an den Präsidenten der Europäischen Kommission aus dem Jahr 2005 zurück. Mit diesem politischen Signal regten die Verfasser (darunter der damalige Bundeskanzler Gerhard Schröder) an, mit dem Aufbau eines öffentlich getragenen Kultur- und Wissensportals, über das die gesamte Bandbreite und Qualität des europäischen Kulturerbes im Internet unentgeltlich und frei von Einflussnahme zugänglich gemacht werden kann, einen Gegenentwurf zu den vielfältigen privatwirtschaftlichen Aktivitäten in diesem Bereich zu schaffen. Dazu sollten auch in den Mitgliedsländern der EU vergleichbare, öffentlich finanzierte Portale entstehen. Die Vorarbeiten zur Errichtung der DDB begannen 2007 mit der Aufforderung der EU-Kommission an alle Mitgliedsstaaten, ein Konzept vorzulegen, wie sie dem europäischen Vorhaben, eine Europäische Digitale Bibliothek (Europeana) aufzubauen, auf nationaler Ebene zuarbeiten werden.

Als nationaler politischer Rahmen für eine Strategie wurden daraufhin von Bund, Ländern und Kommunen „Gemeinsame Eckpunkte zur Errichtung einer Deutschen Digitalen Bibliothek (DDB) als Beitrag zur Europäischen Digitalen Bibliothek“ erarbeitet sowie in einem Verwaltungs- und Finanzabkommen der „Aufbau und Betrieb der Deutschen Digitalen Bibliothek (DDB) als deutscher Bei- 
trag der europäischen Initiative zur Errichtung einer Europäischen Digitalen Bibliothek“, der Europeana, 2009 verbindlich festgelegt.

Die Idee der DDB ist, den kulturellen und wissenschaftlichen Reichtum Deutschlands in seiner ganzen Vielfalt national und international zu präsentieren sowie einen wesentlichen Beitrag zur Förderung der Wissens- und Informationsgesellschaft zu leisten. Konkret wendet sich die DDB sowohl an die allgemeine Öffentlichkeit als auch an Wissenschaftler und an Bildungsvermittler sowie an die Kultur- und Wissenseinrichtungen selbst.

Die DDB hat einen breiten kultur-, wissenschafts- und bildungspolitischen Auftrag, der sich auf vielfältige Bereiche erstreckt:

- Sie dient dem Ziel der gesellschaftlichen Teilhabe an Kultur und Wissen, indem sie zur Sichtbarkeit und Zugänglichmachung von Kultur- und Wissenserbe beiträgt, die Zugangshürden senkt und die Kontextualisierung der Objekte befördert.

- Die DDB bildet ein „Schaufenster“ des deutschen Kultur- und Wissenserbes, das auch nach außen wirkt und Kultur und Wissen aus Deutschland international vermittelt. Sie ist der deutsche Beitrag zur europäischen digitalen Bibliothek Europeana, zu dem sich Deutschland innerhalb der Europäischen Union verpflichtet hat.

- Die elektronische Verfügbarkeit und Präsentation von Objekten aus dem Kultur- und Wissenserbe verbessert schließlich auch die Wahrnehmung und Aufmerksamkeit für die konventionellen (analogen) Angebote der Einrichtungen und macht diese für neue Zielgruppen erfahrbar. Sie verbessert die Möglichkeiten der Partizipation und ermöglicht ganz neue Formen der Kulturund Wissensvermittlung.

- Darüber hinaus stellt die DDB eine Basisinfrastruktur dar, auf deren Grundlage mannigfaltige Anwendungen und Angebote aufsetzen können, die wiederum in spezielle Zielgruppen hineinwirken - etwa im Bereich einzelner Wissenschaftsdisziplinen (vor allem der Geistes- und Kulturwissenschaften) aber auch der schulischen und außerschulischen Bildung. ${ }^{1}$

Für die operative Umsetzung der Ziele wurde 2010 ein interdisziplinäres Netzwerk aus Kultur- und Wissenseinrichtungen eingesetzt. Die Gründungsmitglieder dieses Kompetenznetzwerks (KNW) wurden von der Kultusminis-

1 So ist etwa neben dem bereits bestehenden Archivportal-D die Entwicklung eines nationalen Zeitungsportals und eines Museumsportals auf der Grundlage der DDB-Infrastruktur vorgesehen. terkonferenz, den kommunalen Spitzenverbänden und von der Bundesregierung gemeinsam bestellt. ${ }^{2}$ Das KNW ist Träger der DDB und hat den Auftrag, Aufbau und Betrieb des zentralen nationalen Zugangsportals zu organisieren und die Integration in die Europeana voranzutreiben. Dazu gehören auch die Entwicklung technischer Werkzeuge für die Digitalisierung und Datenhaltung sowie zum erforderlichen Wissensmanagement, die Festlegung von Standards zur Sicherstellung der Kompatibilität und Interoperabilität, die Bereitstellung von allgemeinen Informationen sowie von Informationen zu Aus-/Fortbildungen und Fachveranstaltungen, die Beratung der Kultur- und Wissenseinrichtungen, die Öffentlichkeitsarbeit für die DDB sowie Kooperationen mit Entwicklern von technischen Werkzeugen für Zwecke des Wissensmanagements.

\section{Aktueller Stand}

Inzwischen hat die DDB Ihre Aufbauphase erfolgreich durchlaufen. Das Projekt erreichte mit dem Beta-Launch der Webseite 2012 und mit der Vorstellung der ersten Vollversion 2014 wichtige Meilensteine. Mit gegenwärtig etwa 4000 registrierten Einrichtungen, über 20 Mio. nachgewiesenen Inhalten von mehr als 280 Datenpartnern aus allen Sparten, einer attraktiven Benutzeroberfläche und einer aktiven Lieferbeziehung zur Europeana hat sie ihre wesentlichen Gründungsziele umgesetzt. Bund, Länder und Kommunen wirkten dabei erfolgreich mit den beteiligten Kultur- und Wissenseinrichtungen zusammen, um deren vielfältiges digitales Angebot in einem integrierten Ansatz sicht- und nutzbar zu machen und damit das deutsche Kulturerbe sowohl an interessierte Laien wie an Experten in Deutschland und weltweit zu vermitteln. Die täglich bis zu 13000 Besucher der Webseite (zum Vergleich: ca. 1500 Besucher im Frühjahr 2014) veranschaulichen, dass sich die DDB überdies zu einem in der Öffentlichkeit immer deutlicher wahrgenommenen Angebot entwickelt hat.

2 Dem Kompetenznetzwerk (KNW), das sich im November 2010 auf seiner ersten Mitgliederversammlung konstituierte, gehören derzeit die Bayrische Staatsbibliothek, das Bibliotheksservicezentrum Baden-Württemberg, das Brandenburgische Landesamt für Denkmalpflege und Archäologisches Landesmuseum, das Bundesarchiv, das Deutsche Filminstitut, die Deutsche Nationalbibliothek, das Landesarchiv Baden-Württemberg, das Max-Planck-Institut für Wissenschaftsgeschichte, die Niedersächsische Staats- und Universitätsbibliothek Göttingen, die Sächsische Landesbibliothek - Staats- und Universitätsbibliothek Dresden, die Stiftung Historische Museen Hamburg, die Stiftung Preußischer Kulturbesitz und das Kulturamt der Stadt Düsseldorf an. 
Die steigenden Nutzerzahlen und die wachsenden Zahlen digitalisierter Objekte und Objektnachweise machen deutlich, dass die DDB, die in sie gesetzten Erwartungen, Antworten auf Suchanfragen zeitnah, umfangreich, ohne komplizierte Suchvorgänge, medienübergreifend sowie einrichtungsübergreifend erfüllen kann. Zusätzliche Dienste und Einstiegsmöglichkeiten, die neben den bestehenden Recherchemöglichkeiten entwickelt wurden - wie z. B. virtuelle Ausstellungen, Institutionen- und Personenseiten, Kalenderblatt, Favoritenlisten, Kulturlandkarte sowie die offene Programmierschnittstelle (API) - bieten vielfältige Möglichkeiten und machen die Nutzung und das Arbeiten mit der DDB attraktiv und interessant.

Die DDB ist heute ein spartenübergreifendes und interdisziplinäres Zugangsportal zu den digitalen Angeboten der deutschen Kultur- und Wissenseinrichtungen und macht Kultur und Wissen aus Deutschland national und global zugänglich, sichtbar und erfahrbar. Gleichzeitig vernetzt und kontextualisiert sie die Erschließungsinformationen und öffnet sie für vielfältige Möglichkeiten der Nachnutzung, Anreicherung und Erweiterung.

Nicht ausschließlich auf das Zusammenführen von Daten und das Zugänglichmachen von Inhalten fokussiert, fungiert die DDB auch als Kollaborations- und Serviceplattform. Sie bildet die Infrastruktur für eine interaktive Partizipation auf den unterschiedlichsten Ebenen, vernetzt die Kultur- und Wissenseinrichtungen miteinander und dient der gegenseitigen Unterstützung und dem Austausch von Erfahrungen, Technologien und Diensten. Die DDB bringt innovative Entwicklungen im Bereich der Digitalisierung und Zugänglichmachung voran und trägt maßgeblich zur Standardisierung in diesem Feld bei.

Das Serviceportal DDBpro, ${ }^{3}$ das seit Juli 2015 online ist, wendet sich mit vielfältigen Services und Dienstleistungen insbesondere an die Kultur- und Wissenseinrichtungen. DDBpro ist das Portal für die Datenpartner der DDB und bietet neben Informationen $\mathrm{zu}$ den verschiedenen $\mathrm{An}$ sprechpartnern, Auskünfte und Hinweise zu rechtlichen Fragen, den Leistungen der DDB für die teilnehmenden Einrichtungen zur Datenlieferung und zum API. Bei dieser Schnittstelle handelt es sich um eine öffentliche Programmierschnittstelle, die den maschinellen Zugriff auf Daten ermöglicht und dadurch die Entwicklung vielfältiger Anwendungen erlaubt, die Inhalte der DDB zu nutzen. ${ }^{4}$

$3 \mathrm{https} / / /$ pro.deutsche-digitale-bibliothek.de/.

4 Ein Beispiel für eine Anwendung der API ist das Archivportal-D, https://www.archivportal-d.de/, das erstmals eine deutschlandweite und archivübergreifende Recherche $\mathrm{zu}$ archivischen Erschließungsinformationen und digitalisiertem Archivgut ermöglicht.
Das Serviceportal verfügt auch über eine Selbstbedienungskomponente für Kultureinrichtungen. Sie dient der selbständigen Erfassung und Pflege der eigenen Registrierungsinformationen durch Kultur- und Wissenseinrichtungen (insbesondere Adressangaben und Ansprechpartner), für die Kulturlandkarte der DDB und die Vorbereitung späterer Datenlieferungen.

Darüber hinaus wurde in DDBpro ein Servicebereich mit Links und Downloadmöglichkeiten von allgemeinen und spartenspezifischen Informationen und Dokumenten für die Kultur- und Wissenseinrichtungen angelegt.

Des Weiteren steht nun eine Administrationsoberfläche zur Verfügung, mit der Datensätze von Einrichtungen und Ansprechpartnern geprüft, bearbeitet und freigeschaltet werden können. Der Prozess für die Registrierung und Pflege dieser Datensätze wird somit erheblich vereinfacht, Doppelaufwände und zusätzliche Kommunikationswege entfallen.

Um die spartenübergreifende Sichtbarkeit digitaler Inhalte zu erhöhen und einen komfortablen und unmittelbaren Zugangsweg für die interessierte Öffentlichkeit und Wissenschaft $\mathrm{zu}$ den entsprechenden Beständen und Sammlungen zu weisen, folgt die DDB dem Ansatz, nicht nur digitale Objekte zu zeigen, sondern den Nutzern zusätzliche, darüber hinausweisende Informationen zu präsentieren, die zu vorhandenem, aber (noch) nicht digitalisiertem Kulturgut führen. Die DDB kann so übergreifende Nachweise bieten und nutzt die sich daraus ergebenden Potenziale einer umfassenden semantischen Vernetzung von Erschließungsinformationen. Auf diese Weise eröffnet sie ihren Nutzern über die verbesserte, semantisch angereicherte Navigation zu den digitalen Inhalten auch den $\mathrm{Zu}-$ gang zu Objekten aus deutschen Kultur- und Wissenseinrichtungen, die noch nicht digitalisiert sind. Sie bietet damit, etwa gegenüber der Europeana, die ausschließlich auf bereits digitalisierte Objekte verweist, mit ihrem $\mathrm{Zu}$ gangsportal bereits heute einen echten Zusatznutzen, in dem sie an zentralem Zugangsort bisher nur verteilt erreichbare Informationen zusammenführt, miteinander verknüpft und recherchierbar macht.

Als entscheidender Baustein für die Überführung deutscher Kultur- und Wissensschätze ins digitale Zeitalter hält die DDB das kulturelle Erbe damit im kollektiven Gedächtnis lebendig, auch dann, wenn die Benutzung der Originale, etwa aus konservatorischen Gründen, nicht möglich ist. Zugleich ist sie Katalysator für die vielgestaltigen Bemühungen zu ihrer Digitalisierung und dafür national und international anerkannter Ansprechpartner. 


\section{Kommende Aufgaben und Vorhaben}

Die bisher gewonnenen Erfahrungen zeigen, dass die DDB wichtige gesellschaftliche Bedarfe befriedigt, den mit ihr kooperierenden Einrichtungen eine verbesserte öffentliche Sichtbarkeit verschafft und immer stärkeren Zuspruch bei ihren Nutzern findet. Angesichts der dynamisch wachsenden Nachfrage gilt es nunmehr, den Aufbau der DDB weiter voranzutreiben - insbesondere was die gezielte Erweiterung der Inhalte angeht. Je mehr Objekte und Informationen in der DDB recherchiert werden können, desto attraktiver wird sie für ihre Nutzer und die an ihr beteiligten Kultur- und Wissenseinrichtungen werden. Desto deutlicher werden auch die enormen Potenziale der $\mathrm{Zu}$ sammenführung und semantischen Vernetzung heterogener Daten aus den unterschiedlichsten Kultur- und Wissenseinrichtungen sichtbar.

Der Umfang der über die DDB nachgewiesenen und auffindbaren Sammlungen und Objekte wird daher schrittweise und gezielt erweitert. Auswahl und Priorisierung der Sammlungen richten sich dabei nach definierten, transparenten Kriterien. Die Erweiterung der Inhalte wird sowohl Nachweise digitaler als auch (noch) nicht digitalisierter Sammlungen und Objekte umfassen.

Derzeit ist mit den in der DDB nachgewiesenen Inhalten allerdings erst ein geringer Teil des Kultur- und Wissenserbes über die DDB auffindbar. Dies ergibt sich aus der noch relativ geringen Digitalisierungsquote der Kulturund Wissensschätze in Deutschland sowie aus rechtlichen Hindernissen, insbesondere den Regelungen des geltenden Urheberrechts. Aber auch diejenigen Sammlungen, die digital vorliegen und aus urheberrechtlicher Sicht frei zugänglich gemacht werden könnten, sind bislang nur unvollständig Teil der DDB - etwa weil sie noch gar nicht erfasst wurden, ihre Bearbeitung aus technischen oder rechtlichen Gründen bzw. aufgrund begrenzter Ressourcen nicht möglich war oder sie nicht durch eine „klassische“ Kultur- und Wissenseinrichtung bereitgestellt werden.

Geplante Digitalisierungsvorhaben bzw. laufende Digitalisierungsprojekte werden in Deutschland gegenwärtig nicht an zentralem Ort erfasst, so dass eine Übersicht über vorhandene oder in naher Zukunft für eine Integration in die DDB bereitstehende Inhalte derzeit praktisch unmöglich ist. Die von der DDB geplanten Maßnahmen sollen nun erstmals digital vorliegende und kostenfrei zugängliche Sammlungen und Objekte des Kultur- und Wissenserbes identifizieren und entsprechende Digitalisierungsvorhaben systematisch erfassen, um sie in weiteren Schritten schließlich in die DDB $\mathrm{zu}$ integrieren können. Erhebungen dieser Art sind mit einem hohen Anfangsaufwand verbunden. Dennoch wird sich dieser Aufwand auszahlen, denn mithilfe dieser erfassten Daten kann ein gezielter Bestandsaufbau vorgenommen, können Redundanzen und doppelte Arbeit vermieden werden, eine zuverlässige Planung hinsichtlich der notwendigen Ressourcen durchgeführt und die Integration wichtiger Sammlungen beschleunigt werden.

Parallel dazu wird die Auswahl und Priorisierung potenziell für die DDB relevanter Sammlungen erfolgen, auf transparenten Kriterien basierend und unter Berücksichtigung unterschiedlicher formaler Aspekte - etwa der Größe der Sammlung, der Qualität der digitalen Objekte und der Metadaten sowie ihrer lizenzrechtlichen Verwendbarkeit, der Zielgruppenrelevanz der Inhalte, der Sparte und dem Verhältnis von digitalen Objekten zu reinen Metadaten.

Das Kultur- und Wissenserbe soll möglichst vollständig in der DDB nachgewiesen werden. Deshalb wird die Suche über die unmittelbar in der DDB vorhandenen Daten erweitert bzw. auf alternative Angebote verwiesen und es werden zusätzlich andere Datenquellen (Such-Indizes und externe Datenbanken) integriert.

Dies und die immer weiter zunehmende Anzahl an Datenlieferanten mit ihren individuellen Besonderheiten machen es zugleich notwendig, die Datenlieferungen an die DDB zweckmäßiger und effizienter zu gestalten. Vor diesem Hintergrund spielen die Datenaggregation, -anreicherung und -lieferung eine zunehmend wichtigere Rolle in der Diskussion um eine nachhaltige und leistungsfähige Infrastruktur.

In einem weiteren Schritt ist daher vorgesehen, mit dem Aufbau einer dezentralen Datenlieferinfrastruktur und einem Konzept zur Zusammenarbeit mit Datenaggregatoren, die vorhandene Infrastruktur zu skalieren und bereits digitalisierte Bestände und Sammlungen schneller und effizienter als bisher in die DDB einzubinden. Aggregatoren sind besonders geeignet, die Daten von kleineren und mittleren Einrichtungen zusammenzuführen, zu analysieren, zu harmonisieren, anzureichern und schließlich über definierte Schnittstellen an die DDB zu liefern. So werden die Aufwände für Bearbeitungen der Datenlieferungen und Ingest-Workflows durch die DDB reduziert und der Zuwachs an Daten beschleunigt. Mit einigen Partnern aus dem Kompetenznetzwerk hat die DDB bereits eine Zusammenarbeit aufgebaut, die einer Aggregatorenlösung entspricht. Bei diesen Kooperationen sind DDBspezifische Weiterentwicklungen und betriebliche Aspekte, die Datenaggregation betreffend mit zusätzlichen Aufwänden verbunden, die von den Partnern nicht vollständig übernommen werden können. Daher finanziert die 
DDB diese Aufgaben und Entwicklungen bei den Einrichtungen und wird dies weiter intensivieren, auch um die Umsetzung der von der DDB erarbeiteten Qualitäts- und Servicekriterien sicherzustellen.

Wir wollen erreichen, dass die Kultur- und Wissenseinrichtungen ihre Daten in der bestmöglichen Qualität zur Verfügung stellen. Dazu wird die DDB die Qualitätskriterien für die gelieferten digitalen Informationen schärfen und den Partnern Möglichkeiten eröffnen, eigenständig die Qualität ihrer Daten zu bewerten und sie an die Standards der Deutschen Digitalen Bibliothek anzupassen. Durch die Verwendung standardisierter Schnittstellen und Datenaggregatoren werden Qualitätsverluste bei Datentransfers vermieden und die gelieferten Daten bestmöglich genutzt und in möglichst reichhaltiger Form an Dritte weitergegeben werden.

Um mit den zu erwartenden erheblichen Zuwächsen an Inhalten Schritt zu halten, wird es notwendig sein, den Datendurchsatz bei den Datenprozessen (Transformation, Ingest, Indexierung) zu erhöhen, damit einerseits die steigenden Objekt- und Vorgangszahlen abgewickelt werden können (inkl. der mit steigender Gesamtmenge an Objektnachweisen überproportional wachsenden Update-Vorgängen) und andererseits regelmäßig komplette Re-Ingests $\mathrm{zu}$ ermöglichen. Neben der Beschleunigung der operativen Prozesse müssen die derzeit geltenden betrieblichen Grenzen für die maximale Index- und Repositorygröße so weit nach oben verschoben werden, dass die für die kommenden Jahre zu erwartenden Mengengerüste realisiert werden können.

Die IT-Infrastruktur der DDB basiert zum großen Teil auf einer Systemarchitektur mit Hard- und Softwarekomponenten sowie auf darauf gestützte Betriebsprozesse und Workflows, die ab 2010 entwickelt und implementiert und anschließend schrittweise weiterentwickelt wurden. Um die Zukunftsfähigkeit der Systemarchitektur zu gewährleisten, treibt die DDB 2016 (finanziert durch ein Sonderprojekt des BMI) eine Modernisierung der gesamten ITInfrastruktur voran mit dem Ziel, aktuelle Technologien für die Speicherung, Verwaltung, Anreicherung, Bereitstellung und Auslieferung von Daten sowie zur effizienten Recherche und Navigation zu erproben und sie nach den notwendigen Anpassungen und Erweiterungen für den Produktivbetrieb der DDB zum Einsatz zu bringen.

Schließlich wird es für die DDB auch notwendig sein, Lizenzvereinbarungen (etwa mit den Verwertungsgesellschaften) abzuschließen, um derzeit nicht frei verfügbaren Objekte bzw. Sammlungen - etwa der bildenden Kunst oder der Fotografie (das oft zitierte so genannte ,Schwarze Loch des 20. Jahrhunderts') - für Anwender der DDB ebenfalls zugänglich machen zu können.
Für die Nutzer der DDB ist es wesentlich zu wissen, was sie mit den gefundenen Inhalten tun dürfen und ob sie diese weiterverwenden können. Ziel der DDB ist es daher, alle auffindbaren Inhalte mit einem Hinweis zu den weiteren Nutzungsmöglichkeiten zu versehen. Das Datenmodell sieht vor, dass der Rechtsstatus der digitalen Inhalte und aller Metadaten durchweg gekennzeichnet ist. Alle in der DDB auffindbaren digitalen Inhalte sollen möglichst in einer Version verfügbar sein, die für den Nutzer kostenfrei zugänglich ist und werden einen Hinweis zu den Nutzungsmöglichkeiten enthalten oder mit einer CC (Creative Commons) Lizenz ${ }^{5}$ ausgezeichnet sein. Die zusammengeführten Nachweis- und ErschlieBungsinformationen werden grundsätzlich kostenfrei und in der Regel unter CCO-Bedingungen ${ }^{6}$ (Public Domain Dedication 1.0), also urheberrechtsfreien Bedingungen, bereitgestellt.

\section{Zusammenfassung und Ausblick}

Die Bundesregierung hat in ihrer „Digitalen Agenda 2014-2017“7 die DDB als besonders bedeutsames Projekt hervorgehoben und formuliert,

„Deutschland $\mathrm{zu}$ einem digitalen Kulturland weiter[zu]entwickeln. Dazu gilt es, ein qualitativ hochwertiges Angebot digitaler Inhalte zu sichern. Hierzu werden wir die Rahmenbedingungen für Inhalteanbieter weiter verbessern. Ferner treiben wir die Digitalisierung von Kulturgut weiter voran und verbessern die Zugänglichkeit zum kulturellen und wissenschaftlichen Erbe in Archiven, Bibliotheken und Museen.

- Wir entwickeln eine übergreifende Strategie und Aktionspläne mit geeigneten technischen Lösungen und Standards zur Digitalisierung von Kulturgütern [...] sowie zur langfristigen Bewahrung von Wissen, Informationen und Kulturgütern in digitaler Form und schaffen die dafür notwendigen rechtlichen Rahmenbedingungen.

- Wir stellen - soweit urheberrechtlich zulässig - digitalisierte Kulturgüter und deren Metadaten offen und möglichst unentgeltlich zur Verfügung.

- Wir bauen die ,Deutsche Digitale Bibliothek` weiter auf und aus."

5 Siehe auch http://de.creativecommons.org/.

6 Siehe auch https://www.deutsche-digitale-bibliothek.de/content/1 izenzen-und-lizenzhinweise-rechtssicherheit-der-deutschen-digita len-bibliothek.

7 http://www.digitale-agenda.de/Webs/DA/DE/Home/home_node. html. 
Diesen Zielen der Bundesregierung sieht sich die DDB verpflichtet und innerhalb dieses Rahmens agiert sie: Als von der öffentlichen Hand, gemeinsam von Bund, Ländern und Kommunen getragenes Vorhaben erfüllt die DDB einen langfristigen kultur-, bildungs- und wissenschaftspolitischen Auftrag und schafft bereits heute Nutzen und Mehrwert, in dem sie als Teil einer Gesamtstruktur Inhalte zugänglich macht, miteinander vernetzt und sie für vielfältige Möglichkeiten der Anreicherung, Erweiterung und Nachnutzung öffnet. Sie ist Portal (Rechercheumgebung, Informationsbeschaffung) und sie ist Plattform (Austausch, Diskussion, Partizipation) zugleich und stellt die dazu notwendige technische und organisatorische Infrastruktur bereit. Darüber hinaus ist die DDB der obligatorische deutsche Beitrag zur Europeana, die die Kulturgüter aller Mitgliedstaaten der Europäischen Union bündelt und weltweit zugänglich macht.

Um ihren Auftrag erfüllen und vor den sich daraus ergebenden Herausforderungen bestehen zu können, hat die DDB ihr Leitbild und ihre Strategie 2016 bis $2020^{8}$ veröffentlicht. Sie will die

„zentrale Plattform für Kultur und Wissen in Deutschland [werden], mit der die verteilten Bestände virtuell zusammengeführt und über das Portal als gemeinsamen Zugangspunkt sichtbar gemacht werden. Sie bildet eine vertrauenswürdige Quelle für das Kultur- und Wissenserbe und macht es in attraktiver Weise dauerhaft zugänglich und erfahrbar. Sie öffnet die digitalen Inhalte möglichst weitgehend für die Nachnutzung sowie Anreicherungen und Erweiterungen.“

Die daraus abgeleiteten Aufgabenprioritäten und Maßnahmen für die nächsten fünf Jahre hat die DDB klar formuliert und sollen in den kommenden Jahren vordringlich verfolgt werden:

- Die Erweiterung der Inhalte,

- die Optimierung der Datenprozesse,

- der Ausbau zur Datenplattform,

- die Erhöhung der Nutzerzufriedenheit,

- die Verbesserung der Datenqualität,

- die Erhöhung der Reichweite,

- die Erweiterung des Suchraums.

Die DDB wird in Zukunft aber nur dann erfolgreich sein können, wenn ihr Auf- und Ausbau weiterhin zügig vorangetrieben wird. Die Gewinnung einer stetig wachsenden Zahl mit der DDB kooperierender Einrichtungen und damit die Bereitstellung eines zunehmenden Angebots von Inhalten und ihre Akzeptanz in der Öffentlichkeit

8 https://pro.deutsche-digitale-bibliothek.de/dokumente-und-links. hängen stark von einer kontinuierlichen technischen Weiter- und Fortentwicklung und der Skalierung ihrer Infrastruktur ab. Hierfür sind eine dauerhafte Förderung und eine verbesserte Finanzierungsgrundlage unerlässlich. Dies gilt insbesondere mit Blick auf den dynamischen technischen und kommunikativen Wandel im Onlinebereich, und im Hinblick auf die enorme Komplexität der administrativen und logistischen Anforderungen, die Aufund weiterer Ausbau der DDB stellen werden. Für das Projekt der DDB mit der Vision, das gesamte deutsche Kultur- und Wissenserbe zugänglich und erfahrbar zu machen, gibt es in Deutschland bisher kein Vorbild. Es gilt nunmehr, die DDB angesichts einer stark wachsenden Nachfrage zu etablieren und weiter auszubauen.

Nimmt man das bisher Erreichte und die zukünftige Entwicklung in den Fokus, so wird diese entscheidend davon geprägt sein, inwieweit Bund und Länder den Empfehlungen der 2014 durchgeführten Evaluierung der DDB folgen. Voraussetzung für den weiteren erfolgreichen Aufbau der DDB sind, so das Gutachten, die Überführung der DDB aus dem Projektstatus in eine dauerhaft angelegte Finanzierungs-, Organisations- und Rechtsform sowie eine an der Strategie der DDB orientierte angemessene Anhebung der finanziellen Mittel. Die vielfach als Jahrhundertprojekt bezeichnete Aufgabe der DDB, die Überführung des kulturellen und wissenschaftlichen Erbes Deutschlands in das digitale Zeitalter, hat in der Öffentlichkeit hohe Erwartungen geweckt. Diesem Anspruch wird die DDB nur gerecht werden können, wenn Bund und Länder auch weiterhin gemeinsam an einem Strang ziehen, die entsprechenden Voraussetzungen schaffen und die DDB als dauerhafte staatliche Aufgabe begreifen, um „den Bürgerinnen und Bürgern auch im Netz einen authentischen und umfassenden Zugang zu unserem kulturellen Erbe zu eröffnen." ${ }^{\text {" }}$ Das beinhaltet auch die notwendigen finanziellen Anstrengungen für die Erschließung und die Digitalisierung des kulturellen Erbes.

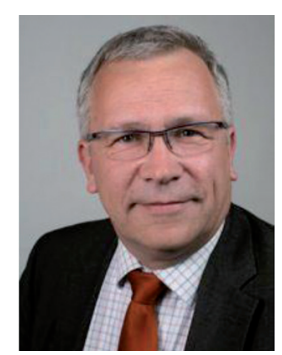

Frank Frischmuth

Deutsche Digitale Bibliothek Geschäftsführer Finanzen, Recht, Kommunikation c/o Stiftung Preußischer Kulturbesitz von-der-Heydt-Str. 16-18 D-10785 Berlin f.frischmuth@hv.spk-berlin.de

9 Grütters, Monika (MdB): Staatsministerin für Kultur und Medien: Rede anlässlich der Eröffnung des Regelbetriebs der Deutschen Digitalen Bibliothek (DDB) in der Gemäldegalerie zu Berlin am 31. März 2014. 